\title{
Milk from cows fed a diet with a high forage:concentrate ratio improves inflammatory state, oxidative stress, and mitochondrial function in rats
}

\author{
Gina Cavaliere, ${ }^{*}$ Giovanna Trinchese, ${ }^{*}$ Nadia Musco, $†$ Federico Infascelli, $\uparrow$ Chiara De Filippo, ${ }^{*}$ \\ Vincenzo Mastellone, $\uparrow$ Valeria Maria Morittu,‡ Pietro Lombardi, $\uparrow$ Raffaella Tudisco, $\uparrow$ Micaela Grossi, $\dagger$ \\ Vincenzo Monda,§ Monica I. Cutrignelli, † Antonietta Messina,§ Serena Calabrò, † Heleena B. Moni, ${ }^{*}$ \\ Luigi Stradella,\# Giovanni Messina, || Marcellino Monda,§ Marianna Crispino, ${ }^{*}$ and Maria Pina Mollica* \\ *Department of Biology, and \\ †Department of Veterinary Medicine and Animal Production, University of Naples Federico II, 80134 Napoli, Italy \\ ‡Department of Health Science, University Magna Graecia of Catanzaro, 88100 Catanzaro, Italy \\ §Department of Experimental Medicine, and \\ \#Department of Gynecology, Obstetric and Reproductive Science, University of Campania "Luigi Vanvitelli," 81100 Caserta, Italy \\ $\|$ Department of Clinical and Experimental Medicine, University of Foggia, 71122 Foggia, Italy
}

\begin{abstract}
Excessive energy intake may evoke complex biochemical processes characterized by inflammation, oxidative stress, and impairment of mitochondrial function that represent the main factors underlying noncommunicable diseases. Because cow milk is widely used for human nutrition and in food industry processing, the nutritional quality of milk is of special interest with respect to human health. In our study, we analyzed milk produced by dairy cows fed a diet characterized by a high forage:concentrate ratio (high forage milk, HFM). In view of the low n-6:n-3 ratio and high content of conjugated linoleic acid of HFM, we studied the effects of this milk on lipid metabolism, inflammation, mitochondrial function, and oxidative stress in a rat model. To this end, we supplemented for 4 wk the diet of male Wistar rats with HFM and with an isocaloric amount $(82 \mathrm{~kJ}, 22 \mathrm{~mL} / \mathrm{d})$ of milk obtained from cows fed a diet with low forage:concentrate ratio, and analyzed the metabolic parameters of the animals. Our results indicate that HFM may positively affect lipid metabolism, leptin:adiponectin ratio, inflammation, mitochondrial function, and oxidative stress, providing the first evidence of the beneficial effects of HFM on rat metabolism.
\end{abstract}

Key words: n-6:n-3 ratio, inflammatory status, redox status, mitochondrial function

\section{INTRODUCTION}

Recently, awareness of the importance of diet to human health has increased. Excessive energy intake,

Received July 23, 2017.

Accepted November 13, 2017.

${ }^{1}$ Corresponding author: mariapia.mollica@unina.it and particularly the excess or inadequate processing of fat in the body, may lead to activation of complex biochemical processes such as inflammation, oxidative stress, and impairment of mitochondrial function (Hernández-Aguilera et al., 2013). These processes are the main factors that underlie aging and noncommunicable diseases, the main types of which are cardiovascular and chronic respiratory diseases, cancers, and diabetes. Indeed, an unhealthy diet often leads to obesity and metabolic disturbances, which have become a serious public health issue worldwide. In this regard, it bears emphasizing that understanding the cellular and molecular mechanisms underlying these metabolic diseases is a crucial step in their prevention and treatment.

Mitochondria, the primary cellular energy-generating system, are known to synthesize key molecules during inflammation and oxidation and thereby serve as the main source of free radicals. Therefore, it is no surprise that mitochondrial dysfunctions are associated with inflammation and other energy-dependent disturbances where cellular oxidative damage is caused by the generation of reactive oxygen species (ROS) exceeding the natural antioxidant activity (Chan, 2006). A growing body of evidence has suggested that a low-grade, chronic inflammatory state may be linked to obesity and its comorbidities, as well as to noncommunicable diseases (Hernández-Aguilera et al., 2013). It is important to underline that the metabolic changes induced by inflammation include alterations in mitochondrial function. Therefore, mitochondrial dysfunction can be both the cause and consequence of inflammatory processes and elicit metabolic adaptations that might be either protective or become progressively detrimental (Currais, 2015).

Various nutritional components are known to modulate the inflammatory state, mitochondrial function, 
and ROS production, thus influencing metabolic homeostasis. To prevent or limit metabolic disorders, special attention should be paid to the choice of appropriate nutritional strategies. The fatty acid profile - in particular, the content of the essential fatty acids n-3 and $\mathrm{n}-6$ - is considered an important parameter to determine the nutritional value of food (Daley et al., 2010). These 2 classes of essential fatty acids, not interconvertible, are metabolically and functionally distinct and often have different physiological functions (i.e., pro- and anti-inflammatory activity for n-6 and n-3, respectively). In particular, a low n-6:n-3 ratio, ranging from 2 to 4, is considered optimal for human health (Simopoulos, 2002). Recent studies have shown that diet is the decisive factor determining the fatty acid profile of cow milk (Sterk et al., 2011); for instance, a high forage:concentrate ratio $(\mathbf{F}: \mathbf{C})$ results in a milk with low n-6:n-3 ratio.

The conjugated linoleic acids are a group of healthy fatty acids. They are positional and geometric isomers derived from octadecadienoic acid, whose content is high in milk fat, and the CLA have been suggested to have immunomodulating, anticarcinogenic, and antiatherosclerosis properties (Dilzer and Park, 2012). The major isomer of CLA, cis-9,trans-11 (rumenic acid), represents up to $80 \%$ of total CLA in food. Ruminant CLA comes from 2 sources: (1) rumen biohydrogenation and (2) endogenous synthesis in the mammary gland and adipose tissue by the activity of stearoylCoA desaturase on trans-11 18:1, the biohydrogenation intermediate of several 18-carbon UFA (Shingfield et al., 2010). The CLA level in the milk from different ruminant species is significantly increased when animals are fed with fresh forage (Jahreis et al., 1997; Kelly et al., 1998; Griinari and Bauman, 1999; Tudisco et al., 2010, 2012, 2014).

Based on this data, some Italian breeders are feeding dairy cows with a high $\mathrm{F}: \mathrm{C}(70: 30)$, which is different from that used in intensive farms (which range from $55: 45$ to $35: 65$ ). By feeding animals a diet with high F:C, milk with a low n-6:n-3 ratio and high CLA level was obtained to satisfy consumer demand for healthy foods (Rubino, 2014).

Several studies have indicated that administration of CLA and n-3 fatty acids to rats improves fatty acid oxidation and decreases inflammation and oxidative stress through the modulation of mitochondrial function (Lionetti et al., 2014; Mollica et al., 2014; Cavaliere et al., 2016). We hypothesized that milk from cows fed a high-forage diet (hereafter, high forage milk, HFM), by modulating mitochondrial function, would ameliorate the inflammatory state and oxidative stress in consumers. To test this hypothesis, we evaluated, in a rat model, the effects of HFM administration on en- ergy balance, lipid metabolism, and anti-inflammatory and antioxidant defenses, compared with those rats fed isoenergetic amounts of milk obtained from cows fed with a diet with a low F:C ratio (low forage milk, LFM).

\section{MATERIALS AND METHODS}

\section{Cow Feeding}

Milk was obtained from a farm located in a hilly area of central Italy (Segni, Rome, Italy; $13^{\circ} 0^{\prime} \mathrm{E}, 41^{\circ} 41^{\prime} \mathrm{N}$, $668 \mathrm{~m}$ above sea level). The farm produced 2 types of commercial milk (LFM and HFM) from Italian Friesian cows ( $\sim 40$ animals for each type of milk) fed 2 different diets (lower or higher F:C ratio, respectively). Ingredients, $\mathrm{F}: \mathrm{C}$ ratio, chemical composition, and nutritive value of the 2 cow diets as well as feed intake are reported in Table 1.

\section{Rat Handling and Feeding}

Male Wistar rats (Charles River, Calco, Lecco, Italy) were individually caged in a temperature-controlled room and exposed to a daily 12-h light/12-h dark cycle with free access to chow and drinking water. Young animals (60 d old; about $350 \mathrm{~g}$ of BW) were used; one group $(\mathrm{n}=7)$ was killed at the beginning of the study to establish baseline measurements. The remaining rats, fed with a standard diet, were divided

Table 1. Diets fed to cows ${ }^{1}$

\begin{tabular}{lcc}
\hline Item & LFM & HFM \\
\hline Diet ingredients (kg as fed) & & \\
Corn silage & 20.0 & - \\
Mixed hay & - & 7.0 \\
Alfalfa hay & 5.0 & 8.5 \\
Wheat bran & 1.6 & 1.0 \\
Corn meal & 3.5 & 3.0 \\
Triticale & 1.5 & 1.0 \\
Fava bean & - & 2.0 \\
Sunflower panel & 1.6 & - \\
Soybean meal & 1.8 & - \\
Forage:concentrate ratio, DM basis & $55: 45$ & $70: 30$ \\
Intake (kg of DM) & 18.9 & 19.3 \\
Chemical composition (g/kg of DM) & 150.0 & 130.0 \\
CP & 29.1 & 18.8 \\
Crude fat & 386.0 & 491.0 \\
NDF & 266.0 & 403.0 \\
ADF & 79.2 & 103.0 \\
ADL & 138.0 & 96.4 \\
Starch & 63.6 & 78.9 \\
Ash & 6.3 & 5.7 \\
NE (MJ/kg) & & \\
\hline
\end{tabular}

${ }^{1}$ LFM and HFM $=$ diets fed to cows to produce low and high forage milk that was later fed to rats in an animal model.

${ }^{2}$ Vicia sativa, Avena sativa, Lolium multiflorum, Trifolium alexandrinum, and Trifolium squarrosum. 
Table 2. Energy intake via chow and milk (kJ; \% of total in parentheses) of rats fed with milk from cows consuming a low forage (LFM) or high forage (HFM) diet

\begin{tabular}{lccr}
\hline Energy intake & Control & LFM-treated & HFM-treated \\
\hline Total energy & 11,899 & 13,975 & 14,010 \\
Chow & 11,899 & $11,968(85.64)$ & $12,003(85.67)$ \\
Milk & & $2,007(14.36)$ & $2,007(14.33)$ \\
\hline
\end{tabular}

into 3 experimental groups $(\mathrm{n}=7$ each): 2 groups were supplemented with equicaloric intakes $(82 \mathrm{~kJ})$ of LFM or HFM $(22 \mathrm{~mL} / \mathrm{d})$ for $4 \mathrm{wk}$; the group that did not receive milk supplement was used as control (the energetic intake of diets is reported in Table 2). After 4 $\mathrm{wk}$, the animals were anesthetized by intraperitoneal injection of chloral hydrate $(40 \mathrm{mg} / 100 \mathrm{~g}$ of $\mathrm{BW})$, and blood was taken from the inferior cava. The liver was removed and subdivided; samples not immediately used for mitochondrial preparation were frozen and stored at $-80^{\circ} \mathrm{C}$. All experiments were conducted in compliance with Italian guidelines for the care and use of research animals.

\section{Analysis of Milk Composition}

Samples of milk (LFM and HFM) were analyzed for protein, fat, and lactose contents by the infrared method using a MilkoScan 133B (Fossomatic, Hillerød, Denmark). In addition, total fat of milk samples was separated using a mixture of hexane:isopropane $(3: 2$, vol/vol; Tudisco et al., 2010). Transmethylation of fatty acids was conducted by a base-catalyzed procedure.

Fatty acid methyl esters were quantified using a GC (ThermoQuest 8000TOP gas chromatograph, equipped with flame-ionization detector; ThermoElectron Corp., Rodano, Milan, Italy) equipped with a CP-SIL 88 fused-silica capillary column $[100 \mathrm{~m} \times 0.25 \mathrm{~mm}$ (internal diameter) with $0.2-\mu \mathrm{m}$ film thickness; Varian, Walnut Creek, CA; Shingfield et al., 2003]. The GC conditions were set as follows: initial oven temperature maintained at $70^{\circ} \mathrm{C}$ for $4 \mathrm{~min}$, increased at $13^{\circ} \mathrm{C} / \mathrm{min}$ to $175^{\circ} \mathrm{C}$ and maintained for $27 \mathrm{~min}$, increased to $215^{\circ} \mathrm{C}$ at $3^{\circ} \mathrm{C} / \mathrm{min}$ and maintained for $38 \mathrm{~min}$, before reverting to $70^{\circ} \mathrm{C}$ at $10^{\circ} \mathrm{C} / \mathrm{min}$. Inlet and detector temperatures were 250 and $260^{\circ} \mathrm{C}$, respectively. The split ratio was 100:1, the helium carrier gas flow rate was $1 \mathrm{~mL} / \mathrm{min}$, the hydrogen flow to the detector was $30 \mathrm{~mL} / \mathrm{min}$, airflow was $350 \mathrm{~mL} / \mathrm{min}$, and the flow of helium make-up gas was $45 \mathrm{~mL} / \mathrm{min}$. Fatty acid peaks were identified using pure standards from Sigma-Aldrich (St. Louis, MO) except CLA cis-9,trans-11 methyl ester, CLA trans-10,cis-12 methyl ester, methyl cis-9, cis-11 octadecadienoate, and methyl trans-9,trans-11 octadecadienoate, which were from Larodan Fine Chemicals $\mathrm{AB}$
(Malmo, Sweden). Fatty acids in samples were identified by comparing the retention times of peaks with that of the standard mixture.

\section{Body Composition, Energy Balance, and Liver Lipid Content}

During treatments, BW and food intake were monitored daily to calculate weight gain and gross energy intake. Spilled food and feces were collected daily for precise calculation of food intake. Energy balance assessments were conducted over 4 wk of treatment by comparative carcass evaluation (Iossa et al., 2002). Metabolizable energy intake was assessed by subtracting the energy measured in feces and urine from gross energy intake, which was determined from the daily food consumption and gross energy density. The gross energy density for the standard diet $(15.8 \mathrm{~kJ} / \mathrm{g})$, and LFM and HFM $(2.70 \mathrm{~kJ} / \mathrm{g})$ as well as the energy density of the feces and the carcasses were determined by bomb calorimetry (adiabatic calorimeter, Parr Instrument Co., Moline, IL). Evaluation of the energy, fat, and protein content in animal carcasses was conducted according to a published protocol (Iossa et al., 2002). Energy efficiency was calculated as the percentage of body energy retained per ME intake, and energy expenditure was determined as the difference between ME intake and energy gain. Total hepatic lipid content was estimated by using the Folch method (Folch et al., 1957).

\section{Oral Glucose Tolerance Test and Insulin Tolerance Test}

For the oral glucose tolerance test, rats were fasted overnight and then orally dosed with glucose $(3 \mathrm{~g} / \mathrm{kg}$ of BW) dissolved in water. For the insulin tolerance test, rats were fasted for $5 \mathrm{~h}$ and then injected intraperitoneally with insulin (rapid-acting homolog, 10 units $/ \mathrm{kg}$ of BW in sterile saline; Novartis, Basel, Switzerland). Blood was collected before the oral glucose and insulin tolerance tests and at various times thereafter, and glucose and insulin levels were determined using a glucose monitor (Brio, Ascensia, NY) calibrated for rats and ELISA (rat insulin; Mercodia, Uppsala, Sweden), respectively. Basal fasting values of serum glucose and insulin were used to calculate the homeostatic model assessment (HOMA) index as follows: [glucose $(\mathrm{mg} /$ $\mathrm{dL}) \times$ insulin $(\mathrm{mU} / \mathrm{L})] / 405$.

\section{Mitochondrial Parameters}

Mitochondrial isolation, oxygen consumption, and proton leakage measurements were performed as previ- 
ously reported (Mollica et al., 2014). Oxygen consumption (polarographically measured using a Clark-type electrode) was measured in the presence of substrates and ADP (state 3 ) or with substrates alone (state 4), and their ratio (respiratory control ratio) was calculated. The rate of mitochondrial fatty acid oxidation was assessed in the presence of palmitoyl-L-carnitine. Mitochondrial proton leakage was assessed by a titration of the steady-state respiration rate as a function of the mitochondrial membrane potential in liver mitochondria. This titration curve is an indirect measurement of proton leakage because the steady-state oxygen consumption rate (i.e., proton efflux rate) in nonphosphorylating mitochondria is equivalent to the proton influx rate due to proton leakage. Carnitinepalmitoyl-transferase (CPT) system and aconitase and superoxide dismutase (SOD) specific activity were measured spectrophotometrically (Flohé and Otting, 1984; Mollica et al., 2014). Rate of mitochondrial $\mathrm{H}_{2} \mathrm{O}_{2}$ release was assayed by following the linear increase in fluorescence caused by the oxidation of homovanillic acid in the presence of horseradish peroxidase (Barja, 1998).

\section{Statistical Analyses}

Data were presented as the means \pm standard errors. Differences among groups were compared by ANOVA followed by the Newman-Keuls post hoc test. Differences were considered statistically significant at $P<$ 0.05. Analyses were performed using GraphPad Prism software (GraphPad Software, San Diego, CA).

\section{RESULTS}

\section{Milk Composition}

Milk protein, fat, and lactose were not significantly affected by cow dietary treatment (Table 3 ). Regarding the fatty acid profile of milk (Table 4), no difference was seen in SFA and MUFA content, whereas C18:3n -3 (linolenic acid) was significantly higher in HFM than in LFM (1.117 vs. $0.23 \%$; $P<0.05)$ as were total n-3 fatty acids (1.212 vs. $0.317 \% ; P<0.05)$ and PUFA (3.615 vs. $2.554 \% ; P<0.05)$. In addition, the $\mathrm{n}-6: \mathrm{n}$

Table 3. Chemical composition of milks from cows consuming a low forage (LFM) or high forage (HFM) diet

\begin{tabular}{lcc}
\hline Item & LFM & HFM \\
\hline Protein (\%) & 3.3 & 3.3 \\
Fat (\%) & 3.5 & 3.7 \\
Lactose (\%) & 4.8 & 4.7 \\
\hline
\end{tabular}

-3 ratio was significantly lower in HFM than in LFM (1.98 vs. $6.96 \% ; P<0.05)$. In contrast, total CLA and cis-9,trans-11 CLA were significantly higher in HFM than in LFM ( 0.79 vs. $0.45 \%$ and 0.73 vs. $0.41 \%$, respectively; $P<0.05)$.

\section{Body Composition and Energy Balance}

As shown in Figure 1, we observed no differences in body composition or energy balance between the LFM and HFM groups. In detail, LFM- and HFM-treated rats exhibited a diminished body water percentage (Figure 1A) and increased body lipids percentage (Figure 1B) and body energy (Figure 1C) compared with controls. No difference in body protein content was observed in the 3 groups of rats (Figure 1D). The LFM-treated animals had increased hepatic lipid content compared with the other 2 groups (Figure 1E). The 2 milk treatments provided similar ME intake, which was significantly higher than that of controls (Figure 1F). The LFMand HFM-treated animals exhibited higher BW, body lipids, and body protein gains compared with control (Figure 1G-I), whereas no difference in energy expenditure was observed in the 3 groups (Figure 1L). Finally, gross energy efficiency was significantly increased in LFM and HFM groups compared with controls (Figure $1 \mathrm{M})$.

\section{Serum Metabolites and Inflammatory Parameters}

Glucose, insulin, triglycerides, cholesterol, alanine aminotransferase, and IL-6 serum levels and HOMA index were not significantly different in the 3 groups of animals (data not shown), indicating that these parameters were not affected by LFM or HFM administration. Confirming the HOMA index results, we did not observe any variation in oral glucose tolerance test or insulin tolerance test (Figure 2G-I). Leptin significantly increased in the LFM and HFM groups compared with control (Figure 2A), whereas adiponectin significantly was decreased in LFM compared with the other 2 groups, and the highest level was found in HFM rats (Figure 2B). Accordingly, the leptin:adiponectin ratio significantly increased in LFM compared with the other 2 groups (Figure 2C). Interestingly, levels of tumor necrosis factor- $\alpha$ (TNF- $\alpha$ ) and IL-1 were significantly decreased in HFM-fed rats compared with controls and LFM-fed rats (Figure 2D, E), indicating a possible antiinflammatory role of HFM. This hypothesis was further supported by the fact that IL-10, an anti-inflammatory cytokine, was found to increase 2 -fold in the LFM group and 2.5-fold in the HFM group compared with controls (Figure 2F). 
Table 4. Fatty acid profile (\%; means \pm SE) of milk from cows consuming a low forage (LFM) or high forage (HFM) diet

\begin{tabular}{|c|c|c|}
\hline Fatty acid $^{1}$ & LFM & HFM \\
\hline C4:0 & $1.194 \pm 0.28$ & $2.121 \pm 0.34$ \\
\hline C6:0 & $1.313 \pm 0.23$ & $1.128 \pm 0.18$ \\
\hline C8:0 & $0.993 \pm 0.20$ & $0.912 \pm 0.12$ \\
\hline C10:0 & $2.522 \pm 0.40$ & $2.449 \pm 0.46$ \\
\hline C11:0 & $0.273 \pm 0.07$ & $0.278 \pm 0.08$ \\
\hline $\mathrm{C} 12: 0$ & $3.067 \pm 0.50$ & $3.211 \pm 0.50$ \\
\hline C13:0 & $0.069 \pm 0.001$ & $0.078 \pm 0.001$ \\
\hline C14:0 & $11.006 \pm 1.03$ & $11.339 \pm 1.10$ \\
\hline C14:1 & $0.000 \pm 0.00$ & $0.299 \pm 0.01$ \\
\hline $\mathrm{C} 15: 0$ & $0.517 \pm 0.01$ & $0.607 \pm 0.01$ \\
\hline C16:0 & $32.825 \pm 2.27$ & $32.987 \pm 2.15$ \\
\hline C16:1 & $1.717 \pm 0.12$ & $2.109 \pm 0.10$ \\
\hline C17:0 & $0.113 \pm 0.02$ & $0.149 \pm 0.01$ \\
\hline C18:0 & $12.049 \pm 1.94$ & $10.546 \pm 1.88$ \\
\hline C18:1n-9 cis & $26.868 \pm 2.90$ & $26.433 \pm 3.01$ \\
\hline C18:1n-9 trans & $0.274 \pm 0.01$ & $0.149 \pm 0.01$ \\
\hline C18:1 trans-10 & $0.109 \pm 0.03$ & $0.120 \pm 0.09$ \\
\hline C18:1 trans-11 & $0.901 \pm 0.08$ & $0.984 \pm 0.05$ \\
\hline C18:2n-6 cis & $1.760 \pm 0.11$ & $1.910 \pm 0.12$ \\
\hline $\mathrm{C} 18: 2 \mathrm{n}-6$ trans & $0.240 \pm 0.01$ & $0.092 \pm 0.02$ \\
\hline C18:3n-6 & $0.178 \pm 0.03$ & $0.192 \pm 0.03$ \\
\hline C18:3n-3 & $0.230 \pm 0.10^{\mathrm{b}}$ & $1.117 \pm 0.11^{\mathrm{a}}$ \\
\hline C20:0 & $0.002 \pm 0.01$ & $0.024 \pm 0.01$ \\
\hline C20:1 & $0.097 \pm 0.01$ & $0.012 \pm 0.01$ \\
\hline $\mathrm{C} 20: 2 \mathrm{n}-6$ & $0.000 \pm 0.00$ & $0.031 \pm 0.01$ \\
\hline C20:3n-3 & $0.012 \pm 0.001$ & $0.016 \pm 0.001$ \\
\hline C20:4n-6 & $0.015 \pm 0.001$ & $0.141 \pm 0.001$ \\
\hline C20:5n-3 & $0.048 \pm 0.002$ & $0.047 \pm 0.001$ \\
\hline $\mathrm{C} 22: 0$ & $0.008 \pm 0.001$ & $0.022 \pm 0.001$ \\
\hline $\mathrm{C} 22: 5 \mathrm{n}-3$ & $0.027 \pm 0.002$ & $0.032 \pm 0.002$ \\
\hline C22:6n-6 & $0.014 \pm 0.001$ & $0.037 \pm 0.001$ \\
\hline SFA & $65.951 \pm 6.23$ & $65.813 \pm 6.17$ \\
\hline MUFA & $28.68 \pm 3.80$ & $28.85 \pm 3.77$ \\
\hline PUFA & $2.554 \pm 0.02^{\mathrm{b}}$ & $3.615 \pm 0.03^{\mathrm{a}}$ \\
\hline$n-3$ & $0.317 \pm 0.01^{\mathrm{b}}$ & $1.212 \pm 0.09^{\mathrm{a}}$ \\
\hline$n-6$ & $2.207 \pm 0.08$ & $2.403 \pm 0.03$ \\
\hline n-6:n-3 & $6.96 \pm 1.00^{\mathrm{A}}$ & $1.98 \pm 0.03^{\mathrm{B}}$ \\
\hline cis-9,trans-11 CLA & $0.41 \pm 0.01^{\mathrm{b}}$ & $0.73 \pm 0.01^{\mathrm{a}}$ \\
\hline trans-10,cis-12 CLA & $0.03 \pm 0.001$ & $0.04 \pm 0.001$ \\
\hline cis-9,cis-11 CLA & $0.01 \pm 0.001$ & $0.02 \pm 0.001$ \\
\hline$\Sigma$ CLA & $0.45 \pm 0.02^{\mathrm{b}}$ & $0.79 \pm 0.02^{\mathrm{a}}$ \\
\hline
\end{tabular}

${ }^{\mathrm{a}, \mathrm{b}}$ Means with different superscript letters are significantly different $(P<0.05)$.

${ }^{\mathrm{A}, \mathrm{B}}$ Means with different superscript letters are significantly different $(P<0.01)$

${ }^{1} \mathrm{SFA}=\mathrm{C} 4: 0+\mathrm{C} 6: 0+\mathrm{C} 8: 0+\mathrm{C} 10: 0+\mathrm{C} 11: 0+\mathrm{C} 12: 0+\mathrm{C} 13: 0+$ $\mathrm{C} 14: 0+\mathrm{C} 15: 0+\mathrm{C} 16: 0+\mathrm{C} 17: 0+\mathrm{C} 18: 0+\mathrm{C} 20: 0+\mathrm{C} 22: 0 ;$ MUFA

$=\mathrm{C} 14: 1+\mathrm{C} 16: 1+\mathrm{C} 18: 1 \mathrm{n}-9$ cis $+\mathrm{C} 18: 1 \mathrm{n}-9$ trans $+\mathrm{C} 20: 1 ;$ PUFA $=\mathrm{C} 18: 2 \mathrm{n}-6$ cis $+\mathrm{C} 18: 2 \mathrm{n}-6$ trans $+\mathrm{C} 18: 3 \mathrm{n}-6+\mathrm{C} 18: 3 \mathrm{n}-3+\mathrm{C} 20: 2 \mathrm{n}-6$ $+\mathrm{C} 20: 3 \mathrm{n}-3+\mathrm{C} 20: 4 \mathrm{n}-6+\mathrm{C} 20: 5 \mathrm{n}-3+\mathrm{C} 22: 5 \mathrm{n}-3+\mathrm{C} 22: 6 \mathrm{n}-6 ; \mathrm{n}-3=$ $\mathrm{C} 18: 3 \mathrm{n}-3+\mathrm{C} 20: 3 \mathrm{n}-3+\mathrm{C} 20: 5 \mathrm{n}-3+\mathrm{C} 22: 5 \mathrm{n}-3: \mathrm{n}-6=\mathrm{C} 18: 2 \mathrm{n}-6$ cis + C18:2n-6 trans + C18:3n-6 + C20:2n-6 + C20:4n-6 + C22:6n-6; CLA $=$ cis-9,trans $-11 \mathrm{CLA}+$ trans-10,cis-12 CLA + cis-9, cis-11 CLA.

\section{Mitochondrial Efficiency and Oxidative Stress}

Mitochondrial state 3 and state 4 respiration, evaluated using succinate and palmitoyl-carnitine as substrates, respectively (to detect fatty acid oxidation), was increased in LFM- and HFM-fed rats compared with the control (Figure 3A, B). Activity of CPT was increased in HFM-fed animals compared with the other
2 groups (Figure 3C). The high quality of mitochondrial preparations was tested by evaluation of respiratory control ratio values (data not shown). Mitochondrial basal and fatty acid-induced proton leakage was increased in LFM- and HFM-treated rats compared with the controls (Figure 3D, E). Finally, the beneficial effects produced by the HFM supplement on liver redox status were clearly indicated by the marked decline in the $\mathrm{H}_{2} \mathrm{O}_{2}$ yield and the significantly increased aconitase and SOD activities (Figure $3 \mathrm{~F}-\mathrm{H}$ ).

\section{DISCUSSION}

In this study, we analyzed the fatty acid profiles of LFM and HFM and surveyed how the differences in milk composition affected metabolic parameters of rats fed with the different milks. Milk from cows fed the higher $\mathrm{F}$ :C ratio (HFM) had a higher, albeit not significant, fat percentage. Digestion of fiber in the rumen produces 2 lipogenic VFA - acetate and butyrate. Butyrate provides energy for the rumen wall, and much of it is converted to BHB in the rumen wall tissue. About half of the fat in milk is synthesized in the udder from acetate and BHB. The other half is transported from the pool of fatty acids circulating in the blood. These can originate from body fat mobilization, absorption from the diet, or from fats metabolized in the liver.

$\alpha$-Linolenic acid (C18:3n-3), total n-3, and CLA were higher in HFM than LFM, confirming previous findings with milk from cows receiving a significant portion of daily DM from pasture and conserved forage-based feeds (Griinari and Bauman, 1999). Fresh forage contains a high percentage of UFA, with $\alpha$-linolenic acid (C18:3) being the predominant n-3 fatty acid (Bergamo et al., 2003). Griinari and Bauman (1999) noted that grazing pasture is a good way of increasing the level of milk PUFA. In our study, C18:3n-3 was higher in HFM, probably because of the higher content of this acid in the cow diet (higher F:C ratio), as suggested by the results obtained in milk from cows (Kelly et al., 1998) and dairy goats (Tsiplakou et al., 2010). Dietary treatment affects milk content of cis-9,trans-11 CLA and total CLA ( $\Sigma \mathrm{CLA}$ ), according to previous findings in goats and sheep (Tsiplakou et al., 2010). The higher concentration of milk CLA found in HFM could be due to the different type of diet, which may influence the rate of microbial fermentation in a way that alters the rate of CLA production or utilization by rumen microbes and, therefore, the concentration of CLA in milk fat (Kelly et al., 1998). Indeed, the higher levels of linoleic and $\alpha$-linolenic acid - the main precursors of cis-9,trans-11 CLA - in the forage could explain the higher CLA content in HFM. 
A

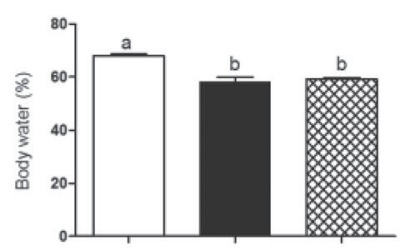

E

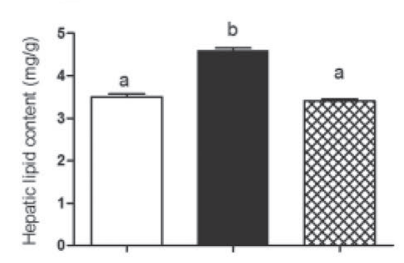

I

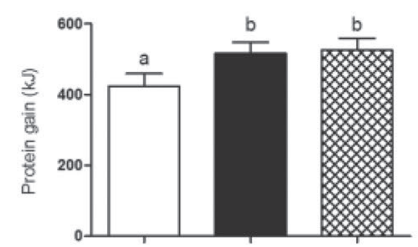

B

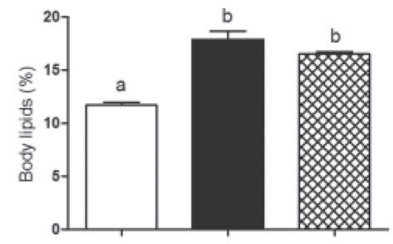

F

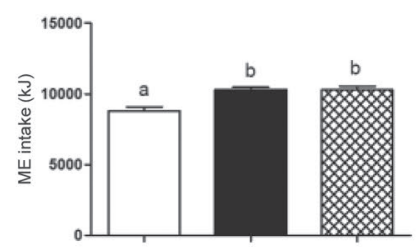

L

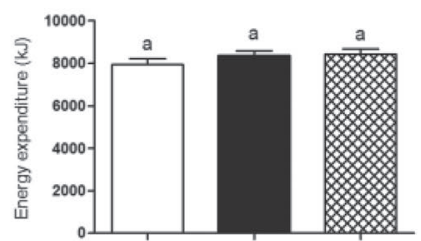

LFM

X HFM

C
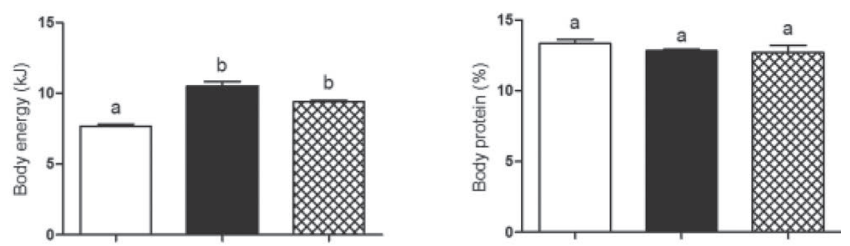

G
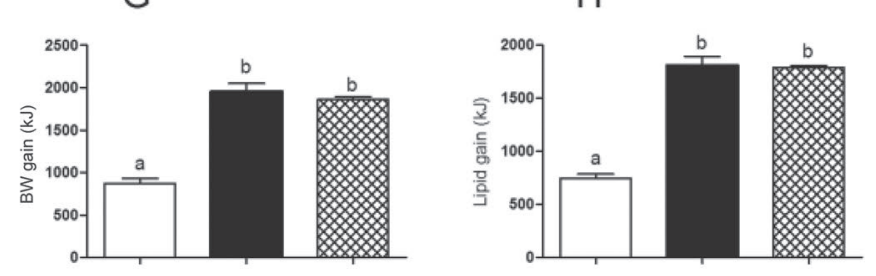

Figure 1. Body composition and energy balance of rats fed with milk from cows consuming a low forage (LFM) or high forage (HFM) diet: (A) water, (B) body lipid, (C) body energy, (D) body protein, (E) hepatic lipid content, (F) energy intake, (G) BW gain, (H) lipid gain, (I) protein gain, (L) energy expenditure, and (M) energy efficiency (means \pm SE; $\mathrm{n}=7$ animals/group). Different letters indicate statistically significant differences between treatment groups $(P<0.05)$.

We hypothesized that the different compositions of LFM and HFM may affect metabolic parameters in the rats. One of our main findings was that the intake of HFM is able to reduce oxidative stress and serum pro-inflammatory cytokines and to increase oxidation of fatty acids in hepatic mitochondria, which eventually reduces fat liver.

Our experiments allowed a comparison of nutritional, immunomodulatory, and antioxidant effects of isoenergetic supplementation with 2 types of cow milk, HFM and LFM. No difference between the HFM and LFM groups was observed in body composition, energy balance, triglycerides, cholesterol, or alanine aminotransferase levels, or in parameters related to glucose homeostasis. Both groups showed an increase in $\mathrm{ME}$ intake and BW gain compared with control rats. The enhanced energy efficiency of LFM- and HFM-fed animals was associated with higher BW and lipid gain compared with control rats. Consistent with increased body lipid level, LFM and HFM groups showed increased levels of leptin compared with controls, and LFM also resulted in a decreased adiponectin level. Surprisingly, the adiponectin level significantly increased in HFMfed rats. Leptin and adiponectin are hormones derived from fat cells that are secreted into the serum, but the leptin level increases with accumulation of fatty mass, whereas adiponectin level decreases (Oda et al., 2008). In some animal models, a decrease in adiponectin level occurred in parallel to decreased insulin sensitivity and preceding the onset of type 2 diabetes (Chakraborti, 2015). Adiponectin secretion is inhibited by several factors, including high level of TNF- $\alpha$ and oxidative stress (Chakraborti, 2015). Therefore, our data, showing an increased adiponectin level in the HFM group associated with decreased levels of TNF- $\alpha$ and $\mathrm{H}_{2} \mathrm{O}_{2}$, may indicate a lower inflammatory state in these animals. It has been proposed that a useful index of metabolic diseases is the leptin:adiponectin ratio, which is better correlated with insulin resistance than the level of leptin or adiponectin alone (Oda, et al. 2008). Our data indicate a significantly higher leptin:adiponectin ratio in LFM than in the control and HFM groups, although LFM animals did not show changes in insulin sensitivity. Further studies, using different amounts of 
milk and administration times, will be required to fully investigate the possible variation of insulin sensitivity in LFM.

The diminished inflammatory state with HFM administration was confirmed by lower levels of TNF- $\alpha$ and IL-1 and an increased level of IL-10 in HFM-fed animals compared with the other 2 groups. Both TNF- $\alpha$ and IL-1 are pro-inflammatory cytokines involved in the progression of metabolic diseases. Conversely, IL-10 is a potent anti-inflammatory cytokine, which drives a negative feedback process during inflammation (Kwilasz et al., 2015). The major roles of TNF- $\alpha$ and other inflammatory cytokines in the progression of metabolic complications are likely to be related to oxidative stress (Rolo et al., 2012). Indeed, ROS and products of lipid peroxidation activate nuclear factor- $\kappa \mathrm{B}$, which induces the synthesis of TNF- $\alpha$ and increases the expression of several pro-inflammatory cytokines, leading to metabolic diseases (Rolo et al., 2012; Hernández-Aguilera et al., 2013). The recognized link among inflammation, redox status, and mitochondrial function prompted us to evaluate the effect of dietary regimens on mitochondrial oxidative capacity and oxidative stress in liver mitochondria in view of the central role played by this organ in energy metabolism. The LFM- and HFM-fed rats exhibited higher hepatic mitochondrial respiratory capacity than did rats in the control group. However, compared with the LFM group, the HFM group exhibited an increased rate of mitochondrial fatty acid oxidation due to enhanced activity of CPT, the rate-limiting enzyme for fatty acid entry into the mitochondria. Thus, the consequent increase in lipid oxidation explains the decreased load of hepatic lipid content found in these rats. Interestingly, compared with the LFM group, in
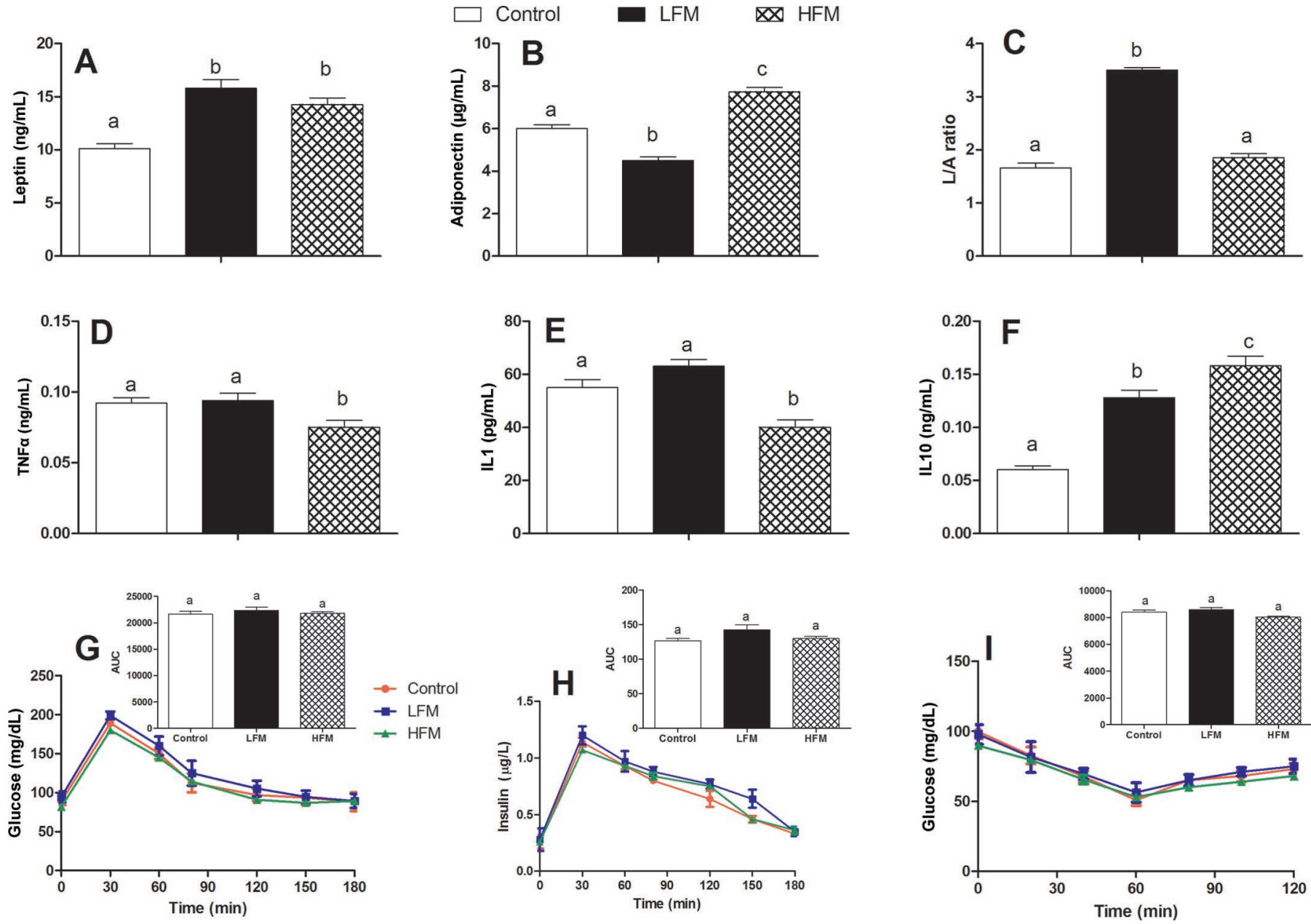

Figure 2. Serum metabolites, inflammatory parameters, and glucose and insulin tolerance test in rats fed with milk from cows consuming a low forage (LFM) or high forage (HFM) diet: (A) leptin (L), (B) adiponectin (A), (C) leptin:adiponectin (L/A) ratio, (D) tumor necrosis factor- $\alpha($ TNF- $\alpha)$, (E) IL-1, (F) IL-10, (G) plasma glucose concentration, (H) plasma insulin concentration at different times after glucose load (oral glucose tolerance test), and (I) plasma glucose concentration at different times after insulin injection (insulin tolerance test). In panels $\mathrm{G}, \mathrm{H}$, and I, the upper inset shows the area under curve (AUC) for each group (mean $\pm \mathrm{SE} ; \mathrm{n}=7$ animals/group). Different letters indicate statistically significant differences between treatment groups $(P<0.05)$. Color version available online. 
the HFM group we observed a reduction of oxidative stress, as indicated by a decrease in $\mathrm{H}_{2} \mathrm{O}_{2}$ production and an increase in activities of aconitase and SOD. A concomitant decline in mitochondrial energy efficiency (thermogenic effect), as evidenced by increased proton leakage, may also contribute to burn fat and reduce mitochondrial oxidative stress parameters. Indeed, an increase in proton leakage was reported as one of the major mechanisms involved in the modulation of membrane potential to control mitochondrial ROS emission (Mailloux and Harper, 2011).

In conclusion, our data provide the first evidence that dietary supplementation with HFM in a rat animal model decreased liver lipid accumulation through an increase in fatty acid oxidation, and decreased inflammation and oxidative stress. The beneficial effects

$\square$ Control $\square$ LFM $\otimes$ HFM
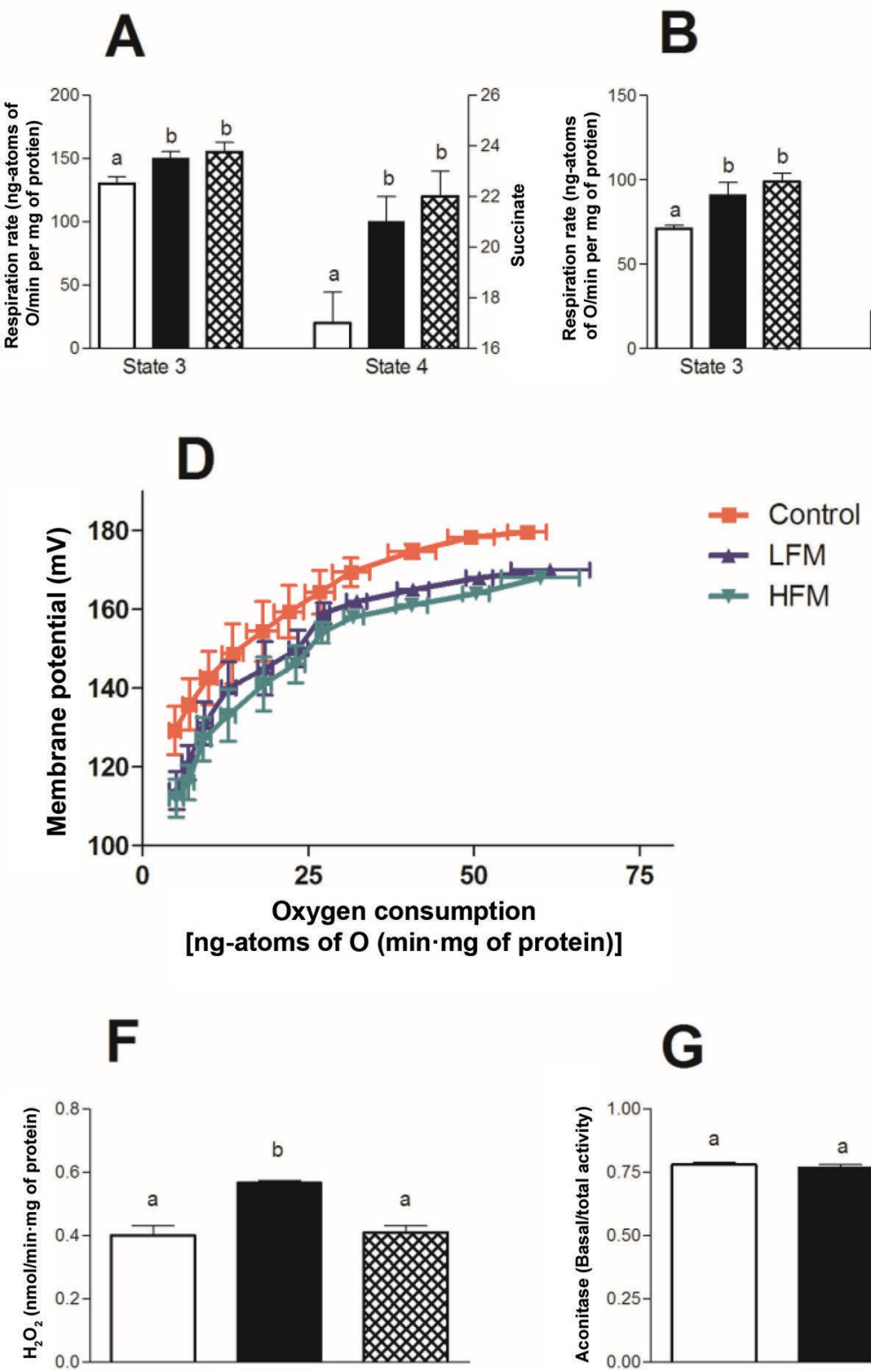
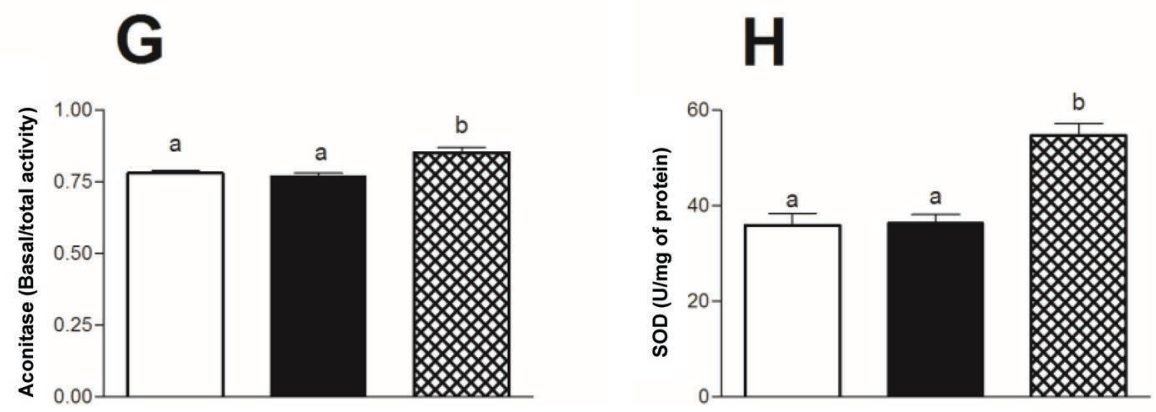

Figure 3. Mitochondrial parameters of rats fed with milk from cows consuming a low forage (LFM) or high forage (HFM) diet: (A and B) hepatic mitochondria respiration rates at states 3 and 4 in the presence of succinate (A) or palmitoyl-carnitine (B) substrates; (C) carnitinepalmitoyl transferase (CPT) activity; (D) basal and (E) fatty acid-induced proton-leakage; (F) intracellular $\mathrm{H}_{2} \mathrm{O}_{2}$ yield, (G) basal aconitase/ total aconitase ratio, and $(\mathrm{H})$ superoxide dismutase (SOD) activity (means $\pm \mathrm{SE}$ from 7 animals/group). Different letters indicate statistically significant differences between treatment groups $(P<0.05)$. Color version available online. 
of HFM are similar to those resulting from n-3 PUFA and CLA intake (Lionetti et al., 2014; Mollica et al., 2014; Cavaliere et al., 2016), allowing us to hypothesize that n-3 PUFA and CLA may be some of the key components of HFM. Further studies will be addressed to verify the specific role of these components.

\section{ACKNOWLEDGMENTS}

The authors thank Roberta Scognamiglio (Department of Biology, University of Naples Federico II, Naples, Italy) for the invaluable technical assistance. Authors Mollica, Infascelli, and Lombardi conceived and designed the experiments; Cavaliere, Trinchese, De Filippo, Tudisco, Grossi, Musco, Cutrignelli, Calabrò, Mastellone, Monda, and Messina performed the experiments; Mollica, Crispino, Infascelli, Lombardi, Morittu, Stradella, and Moni analyzed the data; Mollica, Infascelli, and Lombardi contributed reagents, materials, and analysis tools; Mollica, Infascelli, Lombardi, and Crispino wrote the paper; Tudisco, Messina, and Monda reviewed the manuscript for intellectual content. The authors declare no conflict of interest. The study was funded by Corso di Perfezionamento in Igiene Alimentare, Nutrizione e Benessere.

\section{REFERENCES}

Barja, G. 1998. Mitochondrial free radical production and aging in mammals and birds. Ann. N. Y. Acad. Sci. 854:224-238.

Bergamo, P., E. Fedele, L. Iannibelli, and G. Marzillo. 2003. Fat-soluble vitamin contents and fatty acid composition in organic and conventional Italian dairy products. Food Chem. 82:625-631.

Cavaliere, G., G. Trinchese, P. Bergamo, C. De Filippo, G. Mattace Raso, G. Gifuni, R. Putti, B. H. Moni, R. B. Canani, R. Meli, and M. P. Mollica. 2016. Polyunsaturated fatty acids attenuate diet induced obesity and insulin resistance, modulating mitochondrial respiratory uncoupling in rat skeletal muscle. PLoS One 11:e0149033. https://doi.org/10.1371/journal.pone.0149033.

Chakraborti, C. K. 2015. Role of adiponectin and some other factors linking type 2 diabetes mellitus and obesity. World J. Diabetes 6:1296-1308

Chan, D. C. 2006. Mitochondria: Dynamic organelles in disease, aging, and development. Cell 125:1241-1252.

Currais, A. 2015. Ageing and inflammation-A central role for mitochondria in brain health and disease. Ageing Res. Rev. 21:30-42.

Daley, C. A., A. Abbott, P. S. Doyle, G. A. Nader, and S. Larson. 2010. A review of fatty acid profiles and antioxidant content in grass-fed and grain-fed beef. Nutr. J. 9:10.

Dilzer, A., and Y. Park. 2012. Implication of conjugated linoleic acid (CLA) in human health. Crit. Rev. Food Sci. Nutr. 52:488-513.

Flohé, L., and F. Otting. 1984. Superoxide dismutase assays. Methods Enzymol. 105:93-104.

Folch, J., M. Lees, and G. H. Sloane Stanley. 1957. A simple method for the isolation and purification of total lipids from animal tissue. J. Biol. Chem. 226:497-509.

Griinari, J. M., and D. E. Bauman. 1999. Biosynthesis of conjugated linoleic acid and its incorporation into meat and milk in ruminants. Pages 180-200 in Advances in Conjugated Linoleic Acid Research. Vol. I. M. P. Yurawecz, M. M. Mossoba, J. K. G. Kramer, M. W. Pariza, and G. J. Nelson, ed. AOCS Press, Champaign, IL.
Hernández-Aguilera, A., A. Rull, E. Rodríguez-Gallego, M. RieraBorrull, F. Luciano-Mateo, J. Camps, J. A. Menéndez, and J. Joven. 2013. Mitochondrial dysfunction: A basic mechanism in inflammation-related non-communicable diseases and therapeutic opportunities. Mediators Inflamm. 2013:135698. https://doi.org/ $10.1155 / 2013 / 135698$.

Iossa, S., M. P. Mollica, L. Lionetti, R. Crescenzo, M. Botta, and G. Liverini. 2002. Skeletal muscle oxidative capacity in rats fed highfat diet. Int. J. Obes. Relat. Metab. Disord. 26:65-72.

Jahreis, G., J. Fritsche, and H. Steinhart. 1997. Conjugated linoleic acid in milk fat: High variation depending on production system. Nutr. Res. 17:1479-1484.

Kelly, M. L., E. S. Kolver, D. E. Bauman, M. E. Van Amburgh, and L. D. Muller. 1998. Effect of Intake of pasture on concentrations of conjugated linoleic acid in milk of lactating cows. J. Dairy Sci. 81:1630-1636.

Kwilasz, A. J., P. M. Grace, P. Serbedzija, S. F. Maier, and L. R. Watkins. 2015. The therapeutic potential of interleukin-10 in neuroimmune diseases. Neuropharmacology 96:55-69.

Lionetti, L., M. P. Mollica, I. Donizzetti, G. Gifuni, R. Sica, A. Pignalosa, G. Cavaliere, M. Gaita, C. De Filippo, A. Zorzano, and R. Putti. 2014. High-lard and high-fish-oil diets differ in their effects on function and dynamic behaviour of rat hepatic mitochondria. PLoS One 9:e92753.

Mailloux, R. J., and M. E. Harper. 2011. Uncoupling proteins and the control of mitochondrial reactive oxygen species production. Free Radic. Biol. Med. 51:1106-1115.

Mollica, M. P., G. Trinchese, G. Cavaliere, C. De Filippo, E. Cocca, M. Gaita, A. Della-Gatta, A. Marano, G. Mazzarella, and P. Bergamo. 2014. c9,t11-Conjugated linoleic acid ameliorates steatosis by modulating mitochondrial uncoupling and Nrf2 pathway. J. Lipid Res. 55:837-849.

Oda, N., S. Imamura, T. Fujita, Y. Uchida, K. Inagaki, H. Kakizawa, N. Hayakawa, A. Suzuki, J. Takeda, Y. Horikawa, and M. Itoh. 2008. The ratio of leptin to adiponectin can be used as an index of insulin resistance. Metabolism 57:268-273.

Rolo, A. P., J. S. Teodoro, and C. M. Palmeira. 2012. Role of oxidative stress in the pathogenesis of nonalcoholic steatohepatitis. Free Radic. Biol. Med. 52:59-69.

Rubino, R. 2014. A special section on Latte Nobile: An evolving model. J. Nutr. Ecol. Food Res. 2:214-222

Shingfield, K. J., S. Ahvenjärvi, V. Toivonen, A. Ärölä, K. V. V. Nurmela, P. Huhtanen, and J. M. Griinari. 2003. Effect of dietary fish oil on biohydrogenation of fatty acids and milk fatty acid content in cows. Anim. Sci. 77:165-179.

Shingfield, K. J., L. Bernard, C. Leroux, and Y. Chilliard. 2010. Role of trans fatty acids in the nutritional regulation of mammary lipogenesis in ruminants. Animal 4:1140-1166.

Simopoulos, A. P. 2002. The importance of the ratio of omega-6/ omega-3 essential fatty acids. Biomed. Pharmacother. 56:365-379.

Sterk, A., B. E. Johansson, H. Z. Taweel, M. Murphy, A. M. van Vuuren, W. H. Hendriks, and J. Dijkstra. 2011. Effects of forage type, forage to concentrate ratio, and crushed linseed supplementation on milk fatty acid profile in lactating dairy cows. J. Dairy Sci. 94:6078-6091.

Tsiplakou, E., V. Kotrotsios, I. Hadjigeorgiou, and G. Zervas. 2010. Differences in sheep and goats milk fatty acid profile between conventional and organic farming systems. J. Dairy Res. 77:343-349.

Tudisco, R., S. Calabrò, M. I. Cutrignelli, G. Moniello, M. Grossi, O. J. Gonzalez, V. Piccolo, and F. Infascelli. 2012. Influence of organic systems on stearoyl-CoA desaturase gene expression in goat milk. Small Rumin. Res. 106:S37-S42.

Tudisco, R., M. I. Cutrignelli, S. Calabrò, G. Piccolo, F. Bovera, A. Guglielmelli, G. Moniello, and F. Infascelli. 2010. Influence of organic systems on milk fatty acid profile and CLA in goats. Small Rumin. Res. 88:151-155.

Tudisco, R., M. Grossi, S. Calabrò, M. I. Cutrignelli, N. Musco, L. Addi, and F. Infascelli. 2014. Influence of pasture on goat milk fatty acids and stearoyl-CoA desaturase expression in milk somatic cells. Small Rumin. Res. 122:38-43. 